\title{
English Assistant: A Support Strategy for On-Line Second Language Learning
}

\author{
George R S Weir \\ Department of Computer Science \\ University of Strathclyde \\ Glasgow G1 1XH \\ $U K$ \\ E-mail: gw@cs.strath.ac.uk
}

\author{
Giorgos Lepouras \\ Department of Informatics \\ University of Athens \\ Athens 15771 \\ Greece \\ E-mail:g.lepouras@di.uoa.gr
}

\begin{abstract}
This paper presents an approach to the provision of on-line assistance for learners of English as a second language. The basis for this design is the application of directed support in the form of 'dynamic annotation' to English information. Critically, we aim to provide aid where it is likely to be needed most. Our work falls into two parts. Firstly, we have a mechanism that facilitates the dynamic annotation of English content web pages. Secondly, we are investigating the application of criteria to drive the decision-making that underlies such support.
\end{abstract}

\section{Introduction}

This work seeks to provide a setting in which learners of English as a second language may use Web pages with English content as a basis for language learning. Clearly, an open Web context has considerable potential for selfguided learning. The major overhead is the need to provide learners with support for unfamiliar aspects of the language that they may encounter. If we can secure an environment in which learners are left to browse at will, yet receive appropriate language guidance as annotations to the incoming web content, this should go some way toward the desired facility, without the concurrent presence of dedicated teaching staff.

\section{Language problems}

Language learners face many obstacles to mastery of a second language On the one hand, learners meet words or constructs that are entirely new and unfamiliar. On the other hand, they meet contexts that appear familiar but are not what they seem.

Learners of a similar age and experience inevitably face similar obstacles to comprehension. Not least of their difficulties is the prospect of cross language confusion. Boulton notes four varieties of potential confusion [1]: malapropism, definitional differences, associational differences, and contextual misunderstanding. Additionally, a number of general factors are likely to cause difficulties for non-native users of English. These include 'special use' expressions, (e.g., jargon, slang, or idioms) which are likely to prove unfamiliar or elusive for second-language users.

Naturally, interpretation is coloured by our background and experience. In the context of second-language interpretation such colouring can become interference and lead to error. For example, in a survey of twenty Greek subjects at a Word for Windows seminar for novice users, some people were confused by the term 'font'. Several thought this equivalent to the Greek 'fonto' that means 'background' [2].

Our Web-based learner support seeks to address such scenarios through a combination of dynamic annotation and predefined knowledge-base facility. In addition, we have formulated several criteria as a basis for application of such support.

\section{Annotation facility}

Elsewhere we describe how second language assistance may be given in the context of computer applications [2]. English on-line help information can be supplemented with native language support - a technique that deploys targeted use of second language information, within the context of normal application use, i.e. adjacent to the English original. Beyond predefined local language supplements within Help files, some progress in dynamic second language support has been made for Windowsbased applications. A dynamically accessed database of command terminology has been implemented for use in a PC environment. This allows supplementary Greek terms to be displayed adjacent to their English originals in the context met by the user (as illustrated in Figure 1). 


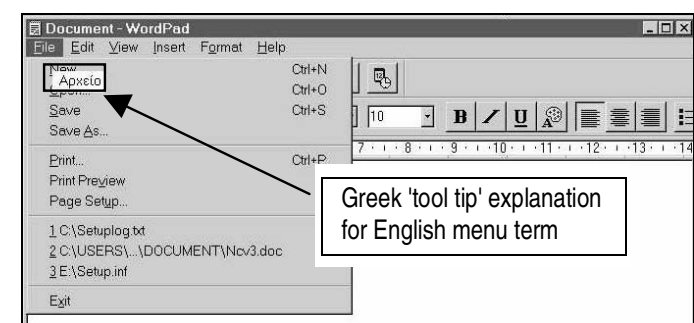

Figure 1 : Dynamic Greek support for menus

Our work touches on two languages that differ significantly from English: Greek and Chinese. Both require font sets that complicate their use in conjunction with English language displays. The Greek case is simpler than Chinese, since fonts are available that combine Greek with the Roman characters used in English. Such technical requirements for establishing native language assistance are prefaced by significant decisions on how to deploy such support. Full translation (e.g., to Chinese) may be given as accompaniment to English Web pages, but this entails significant translation overheads and requires advance selection and preparation of content. Targeted dynamic native language support seems preferable, especially if implemented in an 'open' Web context.

We have developed two approaches to Web-based native language support. The simpler variety uses concurrent frames to supplement English information with local language support. This affords flexibility in the local language support that may be implemented (see Figure 2).

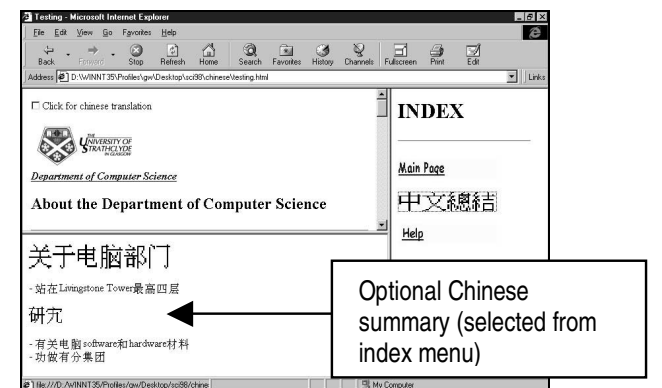

Figure 2 : Frame-based Chinese support

This strategy is limited by the static nature of the second language support. All supplementary support texts must be prepared off-line and added (as frame-based components to Web documents or as associated pop-up definitions) to the original English information. To enhance the application of this support we have implemented a 'look up' system that dynamically checks word contents of the main English text and provides access to local language definitions and explanations.

\section{Dynamic support}

Our initial approach to dynamic support employed an on-line dictionary of informatics developed at the
University of Athens to provide a language reference for English and Greek. Several technical means have been explored toward a dynamic link between this facility and Web page content. An early approach used a Java applet to 'read' terms from the loaded English Web page and seek corresponding Greek explanations from the dictionary. An alternative employs server-side scripts that receive a Uniform Resource Locator from the user, retrieve the document behind the scenes, and perform annotation before sending the result to the user's Web browser. Recently, we have moved to use a proxy server between the learner and the requested Web pages. Here, the user's Web browser is configured to use the proxy server when accessing external Web pages. The proxy requests an external Web page on the user's behalf but does not despatch the received data directly to the user's browser. Instead, the proxy server invokes an external program to scan Web page content for specified key words or phrases.

Based upon the successful matches, an annotated version of the Web page is created 'on the fly' and sent to the local user. In addition, the proxy server may be set to cache the annotated Web pages or the original documents.

The proposed approach to native language annotation promises significant benefits for individual learners. Customisation may be provided across two dimensions: level of language comprehension and modes of annotation. In the first case, the annotation system determines how much and what variety of second language support to add to the source Web pages. This level of support should be determined as a function of the user's English language ability, and based upon weightings attached to items within the annotation database. In the second case, a user may control the manner in which support is attached to the annotated Web pages. According to user wishes, this may use frame-based supplements in the local language, popup second language definitions and explanations, or added Web links to more detailed native language support, including dictionary and thesaurus.

\section{Conclusion}

By combining dynamic annotation techniques with metrics for English language complexity, we see scope to provide a virtual 'English Assistant'. The proposed system extends existing native language support facilities and looks to the plausible application of support criteria as a basis for focused on-line second language support.

\section{References}

[1] Boulton, M., The Anatomy of Language: Saying what we mean, Routledge Kegan Paul, London, 1973.

[2] Weir G.R.S., Lepouras, G. and Sakellaridis U., 'SecondLanguage Help for Windows Applications', HCI'96 Conference on People and Computers XI, Cambridge University Press, 1996. 